\title{
Preoperative Neutrophil-Lymphocyte Ratio for Predicting Surgery-Related Acute Kidney Injury in Non-cardiac Surgery Patients under General Anaesthesia: A Retrospective Cohort Study
}

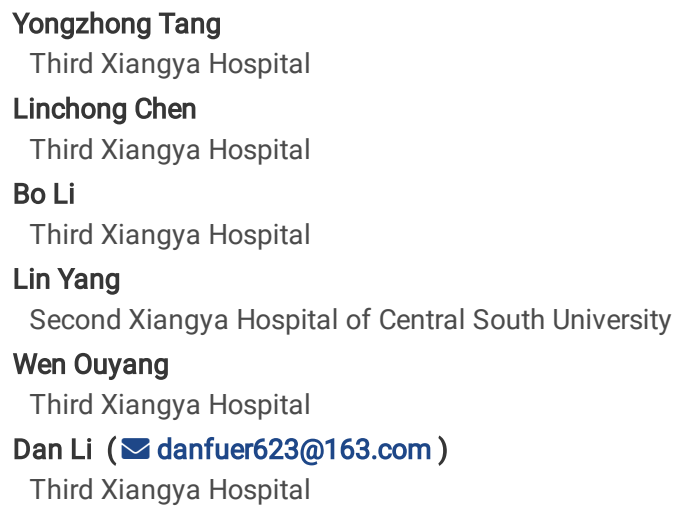




\section{Abstract}

Background This study was conducted to investigate the relationship between the Neutrophil-Lymphocyte Ratio (NLR) and the incidence of surgery-related acute kidney injury (AKI) in non-cardiac surgery patients under general anaesthesia.

Methods In this retrospective study, 5057 patients from Third Xiangya hospital from January 2012 to December 2016 and 1686 patients from Second Xiangya hospital from January 2016 to December 2016 for non-cardiac surgery under general anesthesia were included. According to receiver operating characteristic (ROC) curve constructed by NLR for postoperative AKI囚the cut-off point was obtained as the basis for grouping low or high NLR. The baseline characteristics of two sets were compared with each other. A multi-factor model was constructed by Least absolute shrinkage and selection operator (LASSO) method with the training set, and verified by outside validation set.

Results 243 patients (3.604\%) developed postoperative AKI. The ROC curve showed that the AUC of the NLR for predicting postoperative AKI in non-cardiac surgery was 0.743 (95\% Cl, 0.717-0.769), and the cut-off value was 3.555 (sensitivity, $86.4 \%$; specificity $51.9 \%$ ). There was no significant difference in the baseline characteristics of training set and validation set. The AUC in the training set was $0.817(95 \% \mathrm{Cl}, 0.784-0.850)$, and the AUC in the validation set was $0.804(95 \% \mathrm{Cl}, 0.749-0.858)$, the AUC deviation was $0.012(\mathrm{P}>0.05)$ from validation set, and the likelihood ratio test showed $\mathrm{P}<0.05$.

Conclusion This study showed that preoperative high NLR (NLR $\geq 3.555)$ was an independent risk factor associated with postoperative AKI (OR, $2.410 ; 95 \%$ Cl, 1.371-4.335) in patients for non-cardiac surgery under general anesthesia.

\section{Background}

Acute renal injury (AKI) is a serious postoperative complication with high incidence rate. The incidence of postoperative AKI has been found to range from 3$43 \%[1-4]$, inspite that measures had been taken to protect renal function postoperatively. AKI is also a predictor of long-term prognosis, including chronic kidney disease, end-stage renal disease (ESRD), and cardiovascular disease (heart failure, myocardial infarction), as well as mortality [5, 6]. Currently, markers widely used to evaluate acute renal injury (such as BUN, SCr, and urine volume, etc.) are not sensitive enough to detect early mild impairment of renal function [7]. Other indicators such as Cystatin (CysC), urinary microalbumin (mALB), and 1-microglobulin (1-mg), although more sensitive, are also indicators after renal injury and expensive to carry out in clinical practice. Therefore, the purpose of this study is to explore a cheap $\square$ simple and predictive indexes of postoperative acute renal injury, so as to achieve early prevention, early treatment, and protect the renal function to the maximum extent.

Inflammation is one of the major pathogenic mechanism for AKI [8]. The proportion of neutrophils to lymphocytes, i.e., the neutrophil-lymphocyte ratio (NLR), before surgery is an effective index reflecting inflammation and oxidative stress. A large number of studies have shown that the NLR is closely related to the prognosis of patients with tumors [9, 10], and cardiovascular disease [11-13]. At present, studies have been conducted on NLR and specific high-risk populations of AKI, such as emergency surgery [14], burn surgery [15], cardiac surgery [16], and sepsis patients [17]. However, the relationship between the preoperative NLR and AKI after non-cardiac surgery under general anaesthesia has been barely studied. This retrospective study aimed to assess the significance of the preoperative NLR in predicting acute renal injury in patients for non-cardiac surgery under general anaesthesia, and to obtain a cut-off value of preoperative NLR to distinguish high-risk population and improve the sensitivity of diagnosis.

\section{Methods Patients}

This retrospective study was performed at the Third Xiangya Hospital of Central South University from January 2012 to December $2016(\mathrm{n}=5057)$ and the Second Xiangya Hospital of Central South university from January 2016 to December $2016(n=1686)$. The inclusion criteria were patients aged $\geq 18$ years who underwent non-cardiac surgery under general anesthesia. Patients with a preoperative infection or chronic kidney disease were excluded, along with those admitted for urinary surgery and those with missing data.

This study was in line with the guidelines of the Strengthening of Observational Epidemiological Studies (STROBE) statement, and approved by the ethics committee of the Third Xiangya Hospital of Central South University (2017-S214). Because of observational nature of the study, informed consent was waived by the Third Xiangya Hospital of Central South University.

\section{Data collection}

The following information was collected: 1. Epidemiological data including age and gender; 2 . Preoperative laboratory data including absolute value or percentage of neutrophil and lymphocyte, haemoglobin, platelet distribution width, uric acid, albumin, total bilirubin, creatinine and eGFR calculated using the Chronic Kidney Disease epidemiology collaboration (CKD-EPI) formula [18]. 3. Comorbidities of all the patients (Diabetes, coronary heart disease, peripheral vascular disease and hypertension). 4. Intraoperative data including the operation type (emergency, or laparoscopic), American Society of Anesthesiologists (ASA) grade, operative time, total amount of fluids infusion and blood loss during operation. 5. Postoperative outcomes such as admission to ICU and estimated 10-year survival rate (calculated with Charlson comorbidity index).

\section{Definitions}


Postoperative AKI was defined according to the Kidney Disease: Improving Global Outcomes (KDIGO) 2012 creatinine criteria [19], as one of the following: an increase in serum creatinine by $\geq 0.3 \mathrm{mg} / \mathrm{dL}$ within $48 \mathrm{~h}$ or a $\geq 1.5$-times increase in serum creatinine from baseline within 7 postoperative days. The baseline serum creatinine level was calculated using the lowest level at preoperative day 7 . The NLR was calculated by dividing the absolute neutrophil count by the absolute lymphocyte count. Surgical grade was classified using the surgical classification catalog constituted by the Chinese Ministry of Health, published in 2018.

\section{Statistical Analysis}

IBM SPSS (version 22.0) and R (version 2.12.0) software were used for the statistical analysis. The data are presented as the Median (M) and interquartile range (IQR). Wilcoxon rank sum test was used to compare non-normal distribution continuous variables, and the chi-square test was used to compare the composition ratio of classified data. All $\mathrm{p}$-values $<0.05$ were considered significant.

First, the ROC curve of preoperative NLR was built by R software and the best cut-off value was calculated. Taking the data from The Third Xiangya Hospital as the training set, the variables $\mathrm{P}<0.1$ and some clinical meaningful variables were included in the multi-variable model, and the simplified model was obtained by using the LASSO regression. The data of The Second Xiangya Hospital were taken as the validation set. The ROC curve was drawn to verify that the high preoperative NLR was an associated independent risk factor. Finally, likelihood ratio test was conducted, and the importance of high preoperative NLR was verified again

\section{Results}

In total, 6743 patients were included, of whom 243 (3.604\%) developed postoperative AKI. The ROC curve showed that the AUC of the NLR for predicting postoperative AKI in non-cardiac surgery was 0.743 (95\% $\mathrm{Cl}, 0.717-0.769 ; \mathrm{p}<0.01$ ), and the cut-off value was 3.555 (sensitivity $86.4 \%$; specificity $51.9 \%$ ). It was suggested that preoperative NLR $\geq 3.555$ may associated with increasing the risk of postoperative AKI (Fig. 1).

5057 patients from Xiangya Third Hospital were taken as the training set, and 1686 patients from Xiangya Second Hospital for the validation set. AKI was developed in 176 (3.5\%) and 67 patients (4\%) (Table 1), respectively, in the training set and the verification set. The baseline features of Low and high NLR group were compared in Table 1. However, in order to reduce the size of the variables, the LASSO regression was used. Model I was built with the following risk factors: preoperative high NLR, RBC, lymphocyte absolute value, neutrophil absolute value, platelet distribution width, albumin, total bilirubin, estimated 10year survival rate, emergency, amount of blood loss, and ASA III or IV. The results showed red blood cells, the absolute value of lymphocytes, albumin, and estimated 10 survival rate played a protective role. While Preoperative high NLR was an independent risk factor associated with AKI after non-cardiac surgery (OR, 2.410; 95 \% Cl: 1.371-4.335) (Table 2). 
Table 1

Variables in training set and validation set. Data are shown as mean \pm SD or number (\%), as appropriate. $P=0$ means $P<0.001$, a statistically significant differ AKI, acute kidney injury; CHD, coronary heart disease; DM, diabetes mellitus; ASA, American Society of Anesthesiologists; RBC, red blood cell; Hb, hemoglobin hematocrit; TBIL, total bilirubin; eGFR, estimated glomerular filtration rate.

\begin{tabular}{|c|c|c|c|c|c|c|c|c|}
\hline \multirow[b]{2}{*}{ Variables } & \multicolumn{4}{|c|}{ Training set } & \multicolumn{4}{|c|}{ Validation set } \\
\hline & $P$ & $\begin{array}{l}\text { Low NLR } \\
(N=2556)\end{array}$ & $\begin{array}{l}\text { High NLR } \\
(N=2501)\end{array}$ & $\begin{array}{l}\text { Total } \\
(N=5057)\end{array}$ & $P$ & $\begin{array}{l}\text { Low NLR } \\
(N=849)\end{array}$ & $\begin{array}{l}\text { High NLR } \\
(N=837)\end{array}$ & $\begin{array}{l}\text { Total } \\
(N=1686)\end{array}$ \\
\hline $\begin{array}{l}\text { Postoperative AKI, } \\
n(\%)\end{array}$ & 0 & $24(0.9)$ & $152(6.1)$ & $176(3.5)$ & 0 & $9(1.1)$ & $58(6.9)$ & $67(4.0)$ \\
\hline Male, $n(\%)$ & 0 & $1136(44.4)$ & $1382(55.3)$ & 2518(49.8) & 0 & $388(45.7)$ & $462(55.2)$ & $850(50.4)$ \\
\hline Age, $M(I Q R)$ & 0.245 & $51(44,61)$ & $52(43,62)$ & $52(43,62)$ & 0.304 & $51(43,61)$ & $52(43,62)$ & $51(43,61)$ \\
\hline $\begin{array}{l}\text { Hypertension, } \\
n(\%)\end{array}$ & 0.368 & 1062(41.5) & $1007(40.3)$ & $2069(40.9)$ & 0.219 & $372(43.8)$ & $341(40.7)$ & $713(42.3)$ \\
\hline CHD, $n(\%)$ & 0 & 135(5.3) & $220(8.8)$ & $355(7.0)$ & 0.023 & $42(4.9)$ & $65(7.8)$ & 107(6.3) \\
\hline $\mathrm{DM}, n(\%)$ & 0.023 & $391(15.3)$ & $443(17.7)$ & $834(16.5)$ & 0.181 & $143(16.8)$ & $163(19.5)$ & $306(18.1)$ \\
\hline $\begin{array}{l}\text { Peripheral } \\
\text { vascular disease, } \\
n(\%)\end{array}$ & 0.738 & $241(9.4)$ & 228(9.1) & $469(9.3)$ & 0.809 & $81(9.5)$ & $76(9.1)$ & 157(9.3) \\
\hline ASA grade, $n(\%)$ & 0 & & & & 0 & & & \\
\hline |l|l| & & 1904(74.5) & 1394(55.7) & $3298(65.2)$ & & $641(75.5)$ & $441(52.7)$ & $1082(64.2)$ \\
\hline III & & $614(24.0)$ & 915(36.6) & $1529(30.2)$ & & 198(23.3) & $329(39.3)$ & $527(31.3)$ \\
\hline IV & & $38(1.5)$ & 192(7.7) & $230(4.5)$ & & $10(1.2)$ & $67(8.0)$ & $77(4.6)$ \\
\hline $\begin{array}{l}\text { Estimated } 10 \text {-year } \\
\text { survival rate, } M \\
(I Q R)\end{array}$ & 0.001 & $0.96(0.90,0.98)$ & $0.96(0.78,0.98)$ & $0.96(0.90,0.98)$ & 0.01 & $0.96(0.90,0.98)$ & $0.96(0.78,0.98)$ & $0.96(0.90,0 . \mathrm{C}$ \\
\hline $\mathrm{RBC}, M(I Q R)$ & 0 & $4.3(4.0,4.7)$ & $4.0(3.5,4.5)$ & $4.2(3.7,4.6)$ & 0 & $4.3(4.0,4.7)$ & $4.0(3.5,4.6)$ & $4.2(3.8,4.7)$ \\
\hline $\mathrm{Hb}, M(I Q R)$ & 0 & $128(116,139)$ & $119(101,133)$ & $124(109,137)$ & 0 & $128(115,140)$ & $119(101,136)$ & $125(108,138$ \\
\hline HCT, $M(I Q R)$ & 0 & $\begin{array}{l}39.2 \\
(35.8,42.3)\end{array}$ & $\begin{array}{l}36.3 \\
(31.0,40.5)\end{array}$ & $\begin{array}{l}37.9 \\
(33.5,41.6)\end{array}$ & 0 & $\begin{array}{l}39.3 \\
(35.4,42.6)\end{array}$ & $\begin{array}{l}36.1 \\
(31.5,41.4)\end{array}$ & $\begin{array}{l}37.9 \\
(33.4,42.0)\end{array}$ \\
\hline $\begin{array}{l}\text { Platelet } \\
\text { distribution width, } \\
M(I Q R)\end{array}$ & 0 & $14.3(12.4,16.0)$ & $14.8(12.6,16.2)$ & $14.6(12.5,16.1)$ & 0.027 & $14.3(12.5,16.1)$ & $14.7(12.7,16.2)$ & $14.5(12.6,16$ \\
\hline $\begin{array}{l}\text { Neutrophil } \\
\text { absolute value, } M \\
(I Q R)\end{array}$ & 0 & $3.5(2.8,4.4)$ & $9.1(6.4,12.6)$ & $5.1(3.4,9.2)$ & 0 & $3.5(2.8,4.5)$ & $8.9(6.5,12.5)$ & $5.2(3.5,8.9)$ \\
\hline $\begin{array}{l}\text { Neutrophil } \\
\text { percentage, } M \\
(I Q R)\end{array}$ & 0 & $\begin{array}{l}60.1 \\
(53.8,65.1)\end{array}$ & $\begin{array}{l}84.1 \\
(77.1,89.6)\end{array}$ & $\begin{array}{l}70.5 \\
(59.9,84.0)\end{array}$ & 0 & $\begin{array}{l}60.0 \\
(54.1,65.6)\end{array}$ & $\begin{array}{l}84.2 \\
(77.3,90.1)\end{array}$ & $\begin{array}{l}70.6 \\
(59.8,84.1)\end{array}$ \\
\hline $\begin{array}{l}\text { Lymphocyte } \\
\text { absolute value, } M \\
\text { (IQR) }\end{array}$ & 0 & $1.8(1.5,2.2)$ & $1.0(0.7,1.4)$ & $1.4(1.0,1.9)$ & 0 & $1.8(1.5,2.2)$ & $1.0(0.7,1.3)$ & $1.4(0.9,1.9)$ \\
\hline $\begin{array}{l}\text { Lymphocyte } \\
\text { percentage, } M \\
(I Q R)\end{array}$ & 0 & $\begin{array}{l}29.8 \\
(24.8,35.3)\end{array}$ & $\begin{array}{l}9.4 \\
(5.7,14.5)\end{array}$ & $\begin{array}{l}20.1 \\
(9.5,29.9)\end{array}$ & 0 & $\begin{array}{l}29.5 \\
(25.0,35.1)\end{array}$ & $\begin{array}{l}9.5 \\
(5.6,14.8)\end{array}$ & $\begin{array}{l}20.3 \\
(9.5,29.8)\end{array}$ \\
\hline TBIL, $M(I Q R)$ & 0 & $13.2(10.0,17.6)$ & $15.0(10.7,21.9)$ & $13.9(10.3,19.5)$ & 0 & $13.3(10.0,17.7)$ & $14.9(11.0,21.6)$ & $14.2(10.5,19$ \\
\hline Albumin, $M(I Q R)$ & 0 & $41.2(38.4,44.2)$ & $37.5(32.2,42.0)$ & $39.8(35.6,43.4)$ & 0 & $41.5(38.5,44.4)$ & $37.9(32.6,41.9)$ & $40.0(35.8,43$ \\
\hline $\begin{array}{l}\text { Albumin/Globulin, } \\
M(I Q R)\end{array}$ & 0 & $1.6(1.4,1.8)$ & $1.5(1.3,1.7)$ & $1.5(1.3,1.8)$ & 0 & $1.6(1.4,1.8)$ & $1.5(1.3,1.7)$ & $1.5(1.3,1.7)$ \\
\hline Urea, $M(I Q R)$ & 0.02 & $4.5(3.5,5.5)$ & $4.4(3.2,5.8)$ & $4.4(3.4,5.6)$ & 0.497 & $4.5(3.6,5.6)$ & $4.5(3.3,5.9)$ & $4.5(3.5,5.7)$ \\
\hline Uric acid, $M(I Q R)$ & 0 & $265(209,324)$ & $218(154,290)$ & $245(183,309)$ & 0 & $265(211,334)$ & $222(158,295)$ & $247(183,316$ \\
\hline $\begin{array}{l}\text { Creatinine, } M \\
(I Q R)\end{array}$ & 0.006 & $63(54,74)$ & $62(51,75)$ & $63(53,75)$ & 0.104 & $64(54,76)$ & $63(52,75)$ & $64(53,76)$ \\
\hline eGFR, $M(I Q R)$ & 0 & $103.4(92.7,112.6)$ & $105(93.2,115.7)$ & $104.1(92.9,114.1)$ & 0.034 & $102.9(91.6,112.6)$ & $104.9(92.3,114.9)$ & $103.9(91.8,1$ \\
\hline Emergency, $n(\%)$ & 0 & $160(6.3)$ & $786(31.4)$ & $946(18.7)$ & 0 & $48(5.7)$ & $260(31.1)$ & $308(18.3)$ \\
\hline
\end{tabular}




\begin{tabular}{|c|c|c|c|c|c|c|c|c|}
\hline $\begin{array}{l}\text { Surgical grading, } \\
n(\%)\end{array}$ & & & & & 0.95 & & & \\
\hline 1 & & 1004(39.3) & $902(36.1)$ & 1906(37.7) & & $317(37.3)$ & $307(36.7)$ & $624(37.0)$ \\
\hline 2 & & $1474(57.7)$ & $1515(60.6)$ & 2989(59.1) & & $507(59.7)$ & $504(60.2)$ & $1011(60.0)$ \\
\hline 3 & & $78(3.1)$ & $84(3.4)$ & 162(3.2) & & $25(2.9)$ & $26(3.1)$ & $51(3.0)$ \\
\hline $\begin{array}{l}\text { Laparoscope, } \\
n(\%)\end{array}$ & 0 & $810(31.7)$ & $486(19.4)$ & $1296(25.6)$ & 0 & 261(30.7) & $177(21.1)$ & $438(26.0)$ \\
\hline $\begin{array}{l}\text { Operative time, } M \\
(I Q R)\end{array}$ & 0 & $2.6(1.6,3.7)$ & $2.7(1.8,3.9)$ & $2.7(1.7,3.8)$ & 0.08 & $2.7(1.7,3.8)$ & $2.7(1.9,3.8)$ & $2.7(1.8,3.8)$ \\
\hline $\begin{array}{l}\text { Amount of fluid } \\
\text { infusion, } M(I Q R)\end{array}$ & 0 & $25(16,36)$ & $26(17,36)$ & $26(16,36)$ & 0.21 & $26(16,36)$ & $26(17,36)$ & $26(16,36)$ \\
\hline $\begin{array}{l}\text { Amount of Blood } \\
\text { loss, } M(I Q R)\end{array}$ & 0 & $2.0(0.5,4.0)$ & $3.0(1.0,6.0)$ & $2.0(1.0,5.0)$ & 0 & $2.0(0.8,4.0)$ & $2.5(1.0,5.0)$ & $2.0(1.0,5.0)$ \\
\hline $\begin{array}{l}\text { Admission to ICU, } \\
n(\%)\end{array}$ & 0 & $58(2.3)$ & $365(14.6)$ & $423(8.4)$ & 0 & $9(1.1)$ & 144(17.2) & 153(9.1) \\
\hline
\end{tabular}

Table 2

The result from the lasso regression. $P=0$ means $P<0.001$, a statistically significant difference. Abbreviation: OR (odds ratio), $\mathrm{Cl}$ (confidence interval). RBC (red blood cell) $\left(10^{12} / \mathrm{L}\right)$, absolute values of lymphocytes and neutrophils $\left(10^{9} / \mathrm{L}\right)$, Platelet distribution width ( $\mathrm{fL}$ ), albumin ( $\mathrm{g} / \mathrm{L})$, TBIL (total bilirubin) $(\mu \mathrm{mol} / \mathrm{L})$, blood loss $\left(10^{2} \mathrm{ml}\right)$.

\begin{tabular}{|c|c|c|c|c|c|c|c|c|}
\hline & Risk factor & coefficient & SD & Statistic & $P$ & $O R$ & $2.5 \% \mathrm{Cl}$ & $97.5 \% \mathrm{Cl}$ \\
\hline intercept & - & -3.082 & 0.792 & -3.893 & 0 & 0.046 & 0.01 & 0.216 \\
\hline $\mathrm{X}_{1}$ & Preoperative high NLR & 0.88 & 0.293 & 3.005 & 0.003 & 2.41 & 1.371 & 4.335 \\
\hline$x_{2}$ & $\mathrm{RBC}$ & -0.272 & 0.116 & -2.344 & 0.019 & 0.762 & 0.606 & 0.956 \\
\hline$x_{3}$ & Lymphocyte absolute value & -0.18 & 0.152 & -1.187 & 0.235 & 0.835 & 0.613 & 1.11 \\
\hline $\mathrm{X}_{4}$ & $\begin{array}{l}\text { Neutrophil } \\
\text { absolute value }\end{array}$ & 0.031 & 0.016 & 1.928 & 0.054 & 1.031 & 0.999 & 1.063 \\
\hline$X_{5}$ & Platelet distribution width & 0.083 & 0.027 & 3.03 & 0.002 & 1.086 & 1.028 & 1.145 \\
\hline$x_{6}$ & Albumin & -0.023 & 0.013 & -1.833 & 0.067 & 0.977 & 0.954 & 1.002 \\
\hline$x_{7}$ & TBIL & 0.004 & 0.002 & 2.692 & 0.007 & 1.004 & 1.001 & 1.007 \\
\hline $\mathrm{X}_{8}$ & Estimated 10 -year survival rate & -1.21 & 0.388 & -3.119 & 0.002 & 0.298 & 0.142 & 0.652 \\
\hline$X_{9}$ & Emergency & 0.485 & 0.181 & 2.681 & 0.007 & 1.624 & 1.137 & 2.312 \\
\hline$x_{10}$ & Amount of Blood loss & 0.026 & 0.007 & 3.461 & 0.001 & 1.026 & 1.011 & 1.041 \\
\hline \multirow[t]{2}{*}{$\mathrm{X}_{11}$} & ASA III & 0.75 & 0.194 & 3.857 & 0 & 2.116 & 1.45 & 3.113 \\
\hline & ASA IV & 1.166 & 0.272 & 4.294 & 0 & 3.21 & 1.873 & 5.443 \\
\hline
\end{tabular}

The AUC in the training set was 0.817 (95\% Cl: $0.784-0.85)$, while the validation set was 0.804 (95\% Cl: $0.749-0.85)$, the AUC deviation was 0.012 ( $P=0.68$ > 0.05) (Fig. 2). The validation showed that preoperative high NLR was still an independent risk factor associated with postoperative AKI.

Moreover, the likelihood ratio test is used to verify the established model. Model 1 was established as above, Model II was built the same as Model I but the risk factor preoperative high NLR was removed. The likelihood ratio test showed Model I was significant different with Model II ( $P<0.05)$. This suggest that preoperative high NLR might be of important value in predicting AKI occurrence after non-cardiac surgery.

\section{Discussion}

In our study, the NLR measured on admission, was associated with the development of postoperative AKI in patients who underwent non-cardiac surgery under general anaesthesia. The cut-off value of the NLR was 3.55, the sensitivity of identifying AKI was $86.4 \%$,and the specificity was $51.9 \%$. Preoperative high NLR was an independent risk factor associated with AKI after non-cardiac surgery (OR, 2.410; $95 \%$ Cl: 1.371-4.335).

Inflammation plays an important role in the development of AKI [20]. Some inflammatory marker can predict AKI, such as IL-18 [21], IL-10 [22], IL-6 [22] and a-1 microglobulin [23]. However, these indicators are not carried out as routine examinations in various hospitals; on the other hand, some indicators are only measured postoperatively related to postoperative AKI, which cannot achieve an early warning, so as to carry out intervention in the perioperative period. 
The NLR which can be calculated using data from preoperative blood routine test, is a reliable marker for the systemic inflammatory response. Because the number of neutrophils represents the body's inflammatory response, and the number of lymphocytes represents the body's response to oxidative stress [24]. In the inflammatory response, lymphocytes can be apoptotic, while neutrophils proliferate. Therefore, to a certain extent, the NLR indicates the balance of the inflammatory and anti-inflammatory reactions. A large number of studies have reported that the high NLR is closely related to the development and prognosis of various diseases, such as coronary artery disease, cancer and other diseases [25-27]. In the field of nephorology, Bu et al.[17] reported that NLR value > 17.11 was correlated with septic AKI. Another retrospective study reported by Kim et al.[15] indicated that preoperative NLR value $>11.7$ was associated with early postoperative AKI in burn-injured patients. These two groups of patients are at high risk for postoperative AKI, however, our study included a wider range of patients. By using the optimal cut-off value obtained in our study, the rate of missed diagnosis is lower, which enables clinicians to be more alert to the occurrence of postoperative AKI in patients, strengthen preoperative prevention and management (e.g., avoid the use of nephrotoxic drugs or angiography) and postoperative renal function problems.

The association of preoperative high NLR with postoperative AKI may be the result of neutrophil activation prior to surgery. This, may further lead to endothelial injury and activation of coagulation pathway after surgery, which can stimulate the body to produce inflammatory mediators, induce systemic inflammatory response, and lead to postoperative AKI. Our study focused on the effect of preoperative basic state of patients on postoperative AKI, but also included intraoperative patient conditions, such as hemodynamic issues. There were significant differences in many indicators between the high and low NLR groups, and the factor of $\mathrm{P}<0.1$ was included to correct these indicators. The method of LASSO regression, which is a compression estimation method based on reducing variable set, is adopted to obtain a concise and effective model. A multi-factor regression model (AUC $=0.817)$ was constructed after excluding the interference of age, hypertension, surgical type and other factors. Moreover, through the likelihood ratio validation, it was proved again that preoperative high NLR was an independent risk factor for AKI after non-cardiac surgery, and that preoperative high NLR may have important value in predicting the occurrence of AKI after non-cardiac surgery.

\section{Limitation}

Limitations of this study should also be acknowledged. First, we used the discharge diagnosis to identify diseases, which may not cover all types of CKD patients, including those with minor renal impairment defined by a lower creatinine level. Second, some valuable variables that may be prognostic factors were not evaluated in this study, such as surgical information (operative time and urine output) and inflammatory markers other than the NLR (e.g., cytokines). In view of the above limitations, further research is necessary.

\section{Conclusion}

The present study showed that preoperative high NLR (NLR $\geq 3.555)$ was an independent risk factor for postoperative AKI in patients for non-cardiac surgery under general anesthesia (OR, 2.410; $95 \% \mathrm{Cl}, 1.371-4.335)$. As a simple and rapid indicator, preoperative NLR is valuable in differentiating high-risk groups for postoperative AKI.

\section{Abbreviations}

NLR: Neutrophil-Lymphocyte Ratio; AKI: acute kidney injury.

\section{Declarations}

\section{Ethics approval and consent to participate:}

This study was approved by the ethics committee of the third Xiangya hospital of Central South University (2017-S214). Because of the observational nature of the study, informed consent was waived.

\section{Consent for publication:}

Not applicable.

\section{Availability of data and materials:}

The data used and analyzed in this study are available from the corresponding author on reasonable request.

\section{Competing interests:}

The authors declare they have no competing interests.

\section{Funding:}

This work was supported by the Project of Health and Health Commission of Hunan Province (grant number 20201802), Hunan

Province Key Laboratory Project (grant number 2018TP1009), Natural Science Foundation of Hunan Province邓grant number 2020JJ5854凹and the Research 
and Innovation Funds from Xiangya Bigdata Foundation of Central South University.

\section{Authors' contribution:}

Yongzhong Tang helped in designing the study, analyzing and interpreting the data, and drafting and revising the manuscript. Linchong Chen, Bo Li and Lin Yang helped in collecting, analyzing and interpreting the data. Wen Ouyang helped in Supervising, Funding acquisition and drafting and revising the manuscript. Dan Li helped in designing the study, analyzing and interpreting the data, and drafting and revising the manuscript.

\section{Acknowledgements:}

We thank Dr. Xing Liu for technical consultation.

\section{References}

1. Pickering JW, James MT, Palmer SC. Acute Kidney Injury and Prognosis After Cardiopulmonary Bypass: A Meta-analysis of Cohort Studies. Am J Kidney Dis 2015; 65: 283-93.

2. Marc Najjar MSIG. Acute kidney injury after aortic valve replacement: incidence, risk factors and outcomes. Expert Rev Cardiovasc Ther 2015; 13: 301-16.

3. Kandler K, Jensen ME, Nilsson JC, Moller CH, Steinbruchel DA. Acute kidney injury is independently associated with higher mortality after cardiac surgery. $J$ Cardiothorac Vasc Anesth 2014; 28: 1448-52.

4. Rewa O, Bagshaw SM. Acute kidney injury-epidemiology, outcomes and economics. Nature reviews Nephrology 2014; 10: $193-207$.

5. Horne BD, Anderson JL, John JM, et al. Which white blood cell subtypes predict increased cardiovascular risk? J Am Coll Cardiol 2005; 45: 1638-43.

6. Bhat T, Teli S, Rijal J, et al. Neutrophil to lymphocyte ratio and cardiovascular diseases: a review. Expert review of cardiovascular therapy 2013; 11: 55-9.

7. Wang W, Li Z, Chen Y, Wu H, Zhang S, Chen X. Prediction Value of Serum NGAL in the Diagnosis and Prognosis of Experimental Acute and Chronic Kidney Injuries. Biomolecules 2020; 10.

8. Andrade-Oliveira V, Foresto-Neto O, Watanabe IKM, Zatz R, Camara NOS. Inflammation in Renal Diseases: New and Old Players. Frontiers in pharmacology 2019; 10: 1192.

9. Templeton AJ, McNamara MG, Seruga B, et al. Prognostic role of neutrophil-to-lymphocyte ratio in solid tumors: a systematic review and meta-analysis. Journal of the National Cancer Institute 2014; 106: dju124.

10. Ethier JL, Desautels D, Templeton A, Shah PS, Amir E. Prognostic role of neutrophil-to-lymphocyte ratio in breast cancer: a systematic review and metaanalysis. Breast cancer research: BCR 2017; 19: 2.

11. Durmus G, Belen E, Can MM. Increased neutrophil to lymphocyte ratio predicts myocardial injury in patients undergoing non-cardiac surgery. Heart \& /ung: the journal of critical care 2018; 47: 243-7.

12. Afari ME, Bhat T. Neutrophil to lymphocyte ratio (NLR) and cardiovascular diseases: an update. Expert review of cardiovascular therapy 2016 ; 14 : $573-7$.

13. Zhu N, Zhang D, Wang W, et al. A Novel Coronavirus from Patients with Pneumonia in China, 2019. The New England journal of medicine 2020; 382: 72733.

14. Abu Alfeilat M, Slotki I, Shavit L. Single emergency room measurement of neutrophil/lymphocyte ratio for early detection of acute kidney injury (AKI). Internal and emergency medicine 2018; 13: 717-25.

15. Kim HY, Kong YG, Park JH, Kim YK. Acute kidney injury after burn surgery: Preoperative neutrophil/lymphocyte ratio as a predictive factor. Acta Anaesth Scand 2019; 63: 240-7.

16. Weedle RC, Da Costa M, Veerasingam D, Soo AWS. The use of neutrophil lymphocyte ratio to predict complications post cardiac surgery. Ann Trans/ Med 2019; 7.

17. Bu X, Zhang L, Chen PN, Wu XY. Relation of neutrophil-to-lymphocyte ratio to acute kidney injury in patients with sepsis and septic shock: A retrospective study. International immunopharmacology 2019; 70: 372-7.

18. Matsushita K, Mahmoodi BK, Woodward M, et al. Comparison of risk prediction using the CKD-EPI equation and the MDRD study equation for estimated glomerular filtration rate. Jama 2012; 307: 1941-51.

19. Khwaja A. KDIGO Clinical Practice Guidelines for Acute Kidney Injury. Nephron Clin Pract 2012; 120: C179-C84.

20. Rabb H, Griffin MD, McKay DB, et al. Inflammation in AKI: Current Understanding, Key Questions, and Knowledge Gaps. Journal of the American Society of Nephrology: JASN 2016; 27: 371-9.

21. Parikh CR, Coca SG, Thiessen-Philbrook H, et al. Postoperative Biomarkers Predict Acute Kidney Injury and Poor Outcomes after Adult Cardiac Surgery. Journal Of the American Society Of Nephrology 2011; 22: 1748-57.

22. Zhang WR, Garg AX, Coca SG, et al. Plasma IL-6 and IL-10 Concentrations Predict AKI and Long-Term Mortality in Adults after Cardiac Surgery. Journal of the American Society of Nephrology: JASN 2015; 26: 3123-32.

23. Zhang Y, Zhang B, Wang D, Shi W, Zheng A. Evaluation of Novel Biomarkers for Early Diagnosis of Acute Kidney Injury in Asphyxiated Full-Term Newborns: A Case-Control Study. Medical principles and practice: international journal of the Kuwait University, Health Science Centre 2020; 29: $285-91$.

24. Solak Y, Yilmaz MI, Sonmez A, et al. Neutrophil to lymphocyte ratio independently predicts cardiovascular events in patients with chronic kidney disease. Clinical and experimental nephrology 2013; 17: 532-40. 
25. Unlu M, Balta S, Yildirim AO, Ozturk C, Demir M, Celik T. The Neutrophil-to-Lymphocyte Ratio Is Valuable at All Stages of Coronary Artery Disease. Cardiology 2016; 133: 56.

26. Cicek G, Acikgoz SK, Bozbay M, Altay S, Ugur M, Uluganyan M, Uyarel H. Neutrophil-lymphocyte ratio and platelet-lymphocyte ratio combination can predict prognosis in patients with ST-segment elevation myocardial infarction undergoing primary percutaneous coronary intervention. Angiology 2015; 66: 441-7.

27. Goh BK, Tan DM, Chan CY, et al. Are preoperative blood neutrophil-to-lymphocyte and platelet-to-lymphocyte ratios useful in predicting malignancy in surgically-treated mucin-producing pancreatic cystic neoplasms? Journal of surgical oncology 2015; 112: 366-71.

\section{Figures}

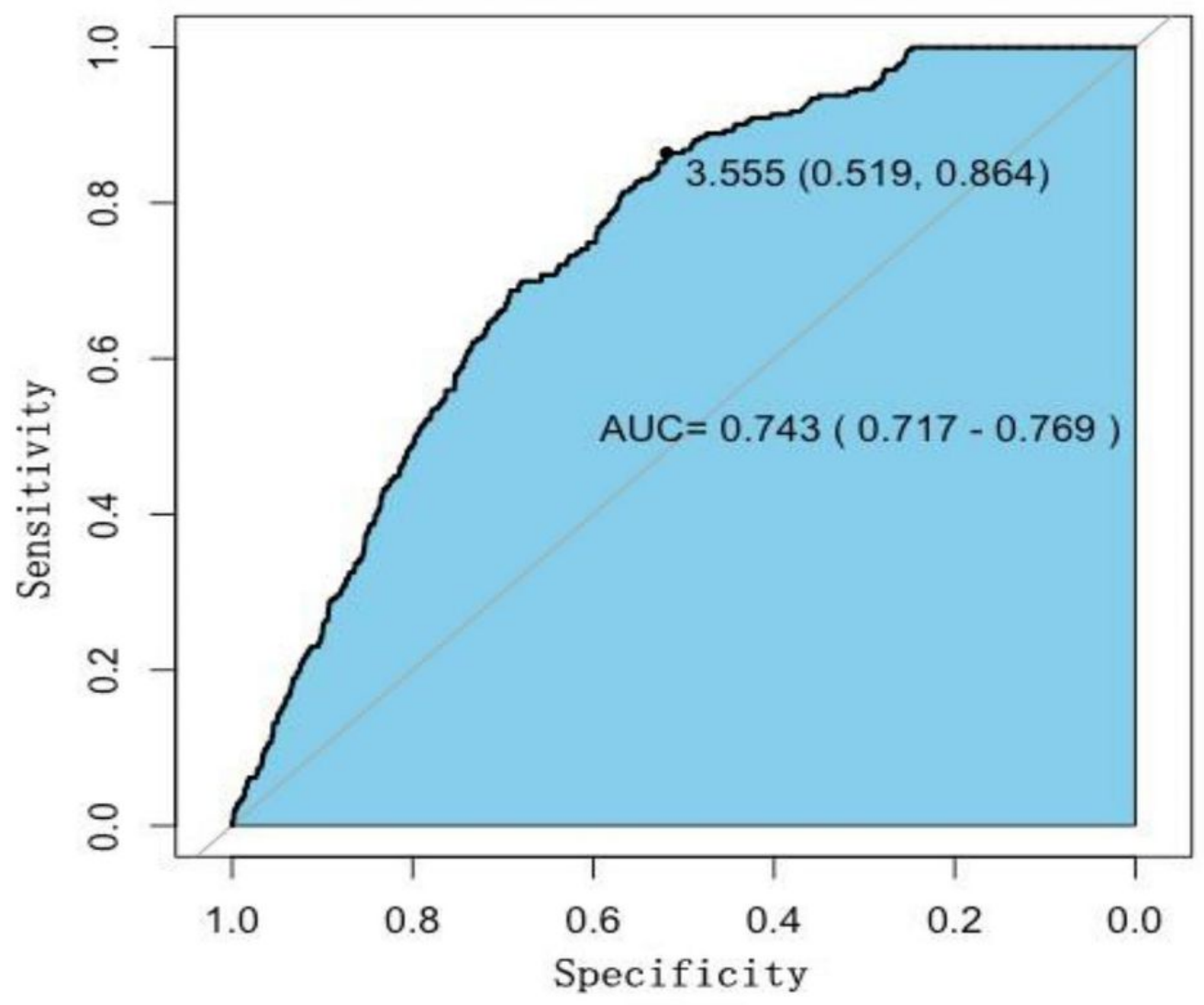

Figure 1

ROC curve analysis of preoperative Neutrophil/lymphocyte ratio for the occurrence postoperative AKI. 


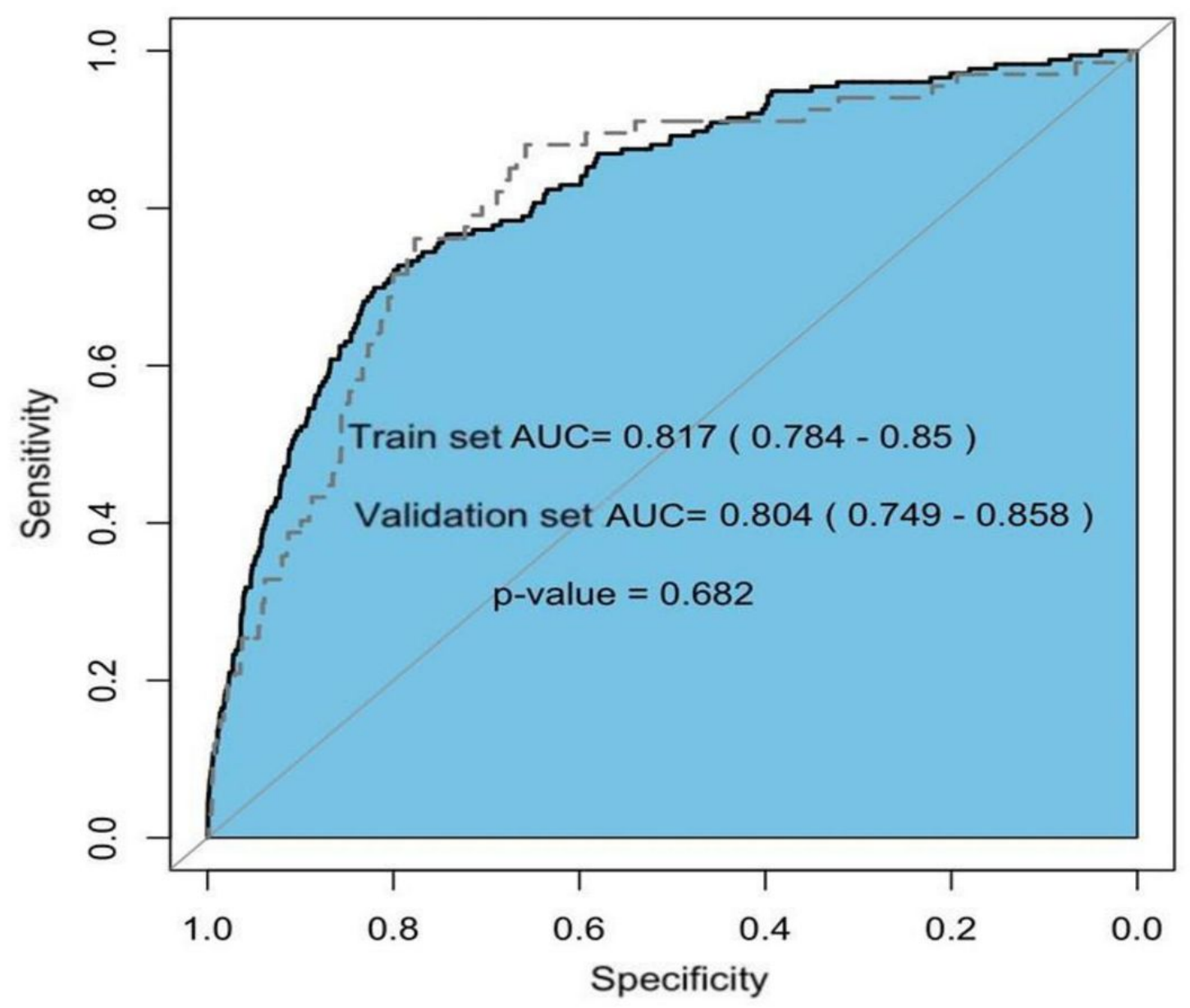

Figure 2

Relationships between the ROC of training set and validation set 